\title{
Mechanical Parameters Effects on Acoustic Absorption at Polymer Foam
}

\author{
Lyes Dib, ${ }^{1}$ Samia Bouhedja, ${ }^{1,2}$ and Hamza Amrani1 \\ ${ }^{1}$ Hyperfrequencies and Semiconductor Laboratory, Faculty of Sciences of Technology, Mentouri Brothers University of Constantine, \\ P.B. 325, 25017 Constantine, Algeria \\ ${ }^{2}$ Faculty of Medical Science, Constantine 3 University, P.B. 125, 25000 Constantine, Algeria
}

Correspondence should be addressed to Lyes Dib; lyes19801@hotmail.com

Received 20 June 2015; Revised 9 August 2015; Accepted 10 August 2015

Academic Editor: Belal F. Yousif

Copyright (C) 2015 Lyes Dib et al. This is an open access article distributed under the Creative Commons Attribution License, which permits unrestricted use, distribution, and reproduction in any medium, provided the original work is properly cited.

\begin{abstract}
Polymer foams have acoustic absorption properties that play an important role in reducing noise level. When the skeleton is set to motion, it is necessary to use generalized Biot-Allard model which takes into account the deformation of the skeleton and the fluid and the interactions between them. The aim of this work is to study the quality of acoustic absorption in polyurethane foam and to show the importance of the structural vibration of this foam on the absorption by varying mechanical parameters (Young's modulus $E$, Poisson's coefficient $\nu$, structural damping factor $\eta$, and the density $\rho_{1}$ ). We calculated the absorption coefficient analytically using classical Biot formulation $\left(u^{s}, u^{f}\right)$ and numerically using Biot mixed formulation $\left(u^{s}, p\right)$ in 3D COMSOL Multiphysics. The obtained results are compared together and show an excellent agreement. Afterwards, we studied the effect of varying each mechanical parameter independently on the absorption in interval of $\pm 20 \%$. The simulations show that these parameters have an influence on the sound absorption around the resonance frequency $f_{r}$.
\end{abstract}

\section{Introduction}

Porous materials are materials well known for their promising applications in many areas, for example, in automotive and aeronautics; they are mainly used to reduce noise level. According to their frame state, they can be classified to three types: elastic, rigid, or limp. Metallic foams and fiber layers are common examples of materials having limp or rigid frame, respectively. Because of the huge rigidity in their frames, only longitudinal waves can propagate inside the fluid phase. The "equivalent fluid" model is often used to model these types of materials [1]. This model is characterized by the effective density and the compression modulus. Many works have been done to evaluate these effective properties to predict the behavior of these types of materials [2-7]. Polymer foams (polyurethane) are well known porous materials with elastic frame. In a poroelastic medium, acoustic wave propagation is described by a generalized Biot-Allard model [8-10]. Unlike rigid or limp materials, waves can propagate in both phases of the poroelastic medium, that is, a longitudinal acoustic wave in the fluid phase and both longitudinal and transversal waves in the solid phase.

In this work, we are interested in studying the acoustic behavior of poroelastic materials (polymer foams), specifically polyurethane. To know the quality of absorption of this material, it is necessary to have all the properties that define every phase, that is, fluid and solid, and the interconnection between them and to know other parameters that can influence this quality. A lot of works have been done to study these properties, such as the porosity, resistivity, tortuosity, and viscous and thermal characteristic lengths [11-13].

In this paper, we calculate the surface impedance and the absorption coefficient versus frequency, and we study the influence of the mechanical parameters on the quality of the absorption. We use the classical $\left(u^{s}, u^{f}\right)$ formulation of Biot-Allard model for the analytical calculations and a combination of $\left(u^{s}, p\right)$ formulation and finite element approach for the numerical calculation using COMSOL environment $[14,15]$. 


\section{Modeling of Sound Absorbing Materials}

In this section, we will present two important formulations, which with we can study and predict the acoustic behavior of poroelastic medium. These formulations are the classic formulation of Biot known as displacement-displacement formulation $\left(u^{s}, u^{f}\right)$ which uses 6 variables for $3 \mathrm{D}$ space, 3 for solid phase and 3 for fluid phase, and a mixed formulation that uses 4 space variables; it had been developed by Atalla et al. [16]. This formulation is used to describe the fluid phase and the acoustic pressure $p$ in the pores. It is called displacement-pressure formulation $\left(u^{s}, p\right)$.

2.1. The $\left(u^{s}, u^{f}\right)$ Formulation for Poroelastic Material. For a monochromatic acoustic wave, of pulsation $\omega$, incident on porous medium with elastic structure, the wave equation in the solid skeleton, and saturating fluid are obtained from the energetic considerations [1]. With the conventional $e^{+j \omega t}$, the equations can be written as follows:

(i) In the solid phase,

$$
\begin{gathered}
N \nabla^{2} u^{s}+(P-N) \nabla \nabla u^{s}+Q \nabla\left(\nabla u^{f}\right) \\
+\omega^{2}\left(\tilde{\rho}_{11} u^{s}+\tilde{\rho}_{12} u^{f}\right)=0 .
\end{gathered}
$$

(ii) In the fluid phase,

$$
Q \nabla\left(\nabla u^{s}\right)-R \nabla\left(\nabla u^{s}\right)+\omega\left(\tilde{\rho}_{12} u^{s}+\tilde{\rho}_{22} u^{f}\right)=0 .
$$

$u^{s}$ and $u^{f}$ are, respectively, the displacement vector in the structure and the macroscopic displacement vector in the saturating fluid. The coefficients, $\widetilde{\rho}_{11}, \widetilde{\rho}_{12}$, and $\widetilde{\rho}_{22}$ in (3), are defined from mass coupling factors, $\rho_{11}, \rho_{12}$, and $\rho_{22}$ in (4), and from viscous coupling parameter $b$ in (5). Consider

$$
\begin{aligned}
& \tilde{\rho}_{11}=\rho_{11}-j \frac{b}{\omega}, \\
& \tilde{\rho}_{12}=\rho_{12}+j \frac{b}{\omega}, \\
& \tilde{\rho}_{22}=\rho_{22}-j \frac{b}{\omega} .
\end{aligned}
$$

Mass coupling factors [17] are as follows:

$$
\begin{aligned}
& \rho_{11}=\rho_{1}-\rho_{22}, \\
& \rho_{12}=-\phi \rho_{0}\left(\alpha_{\infty}-1\right), \\
& \rho_{22}=\phi \rho_{0}-\rho_{12} .
\end{aligned}
$$

Viscous coupling parameter [18] is as follows:

$$
b=\sigma \phi^{2}\left(1+\frac{j 4 \alpha_{\infty}^{2} \eta \rho_{0} \omega}{\sigma^{2} \wedge^{2} \phi^{2}}\right)^{1 / 2} .
$$

$\phi$ is the porosity of the considered medium. The elasticity coefficients $P, N, Q$, and $R$ introduced by Biot model are evaluated by three Gedenken experiments [1]. In the case where the material which composes the solid skeleton is less compressible, the four elasticity coefficients can be approximated by the following relations:

$$
\begin{aligned}
P & =\frac{4}{3} N+K_{b}+\frac{(1-\phi)^{2}}{\phi} K_{f}, \\
N & =\frac{3 K_{b}(1-2 \nu)}{2(\nu+1)}, \\
Q & =(1-\phi) K_{f}, \\
R & =\phi K_{f} .
\end{aligned}
$$

$K_{b}$ is the compressibility modulus of the solid frame (in vacuum) which can be evaluated by formulation (7) [1]. $K_{f}$ is the compressibility modulus of the fluid contained in the material pores and corresponds to the dynamic compressibility established for the equivalent fluid (porous material with rigid frame) in the Johnson et al. model [3] (8). Consider

$$
\begin{aligned}
K_{b} & =\frac{E}{3(1-2 \nu)}, \\
K_{f} & =\frac{\gamma P_{0}}{\gamma-(\gamma-1) H_{f}}, \\
H_{f} & =\frac{1}{1+\left(8 \eta / j \wedge^{\prime 2} \rho_{0} P_{r} \omega\right)\left(1+j \wedge^{\prime 2} \rho_{0} P_{r} \omega / 16 \eta\right)^{1 / 2}} .
\end{aligned}
$$

$E$ and $\nu$ are, respectively, Young's modulus and the Poisson coefficient of the deformable solid matrix. $\sigma, \alpha_{\infty}$, and $\wedge$ are the characteristic parameters of the studied porous medium. $\rho_{1}$ represents the density of the solid frame and $\rho_{2}$ is the effective density defined in the case of the equivalent fluid (10). Consider

$$
\begin{aligned}
\rho_{2} & =\alpha_{\infty} \rho_{0}\left[1-\frac{j \sigma \phi G_{\omega}}{\rho_{0} \alpha_{\infty} \omega}\right] \\
G_{\omega} & =\left(1+\frac{4 j \alpha_{\infty}^{2} \eta \rho_{0} \omega}{\sigma^{2} \phi^{2} \wedge^{2}}\right)^{1 / 2} .
\end{aligned}
$$

2.2. Acoustic Wave Propagation. Two categories of waves propagate at a time across the solid skeleton of the material and in the saturating fluid inside the pores: compression waves and shear waves. In order to determine the characteristics associated to these two types of waves, the displacement vectors $u^{s}$ and $u^{f}$ are replaced in (1) and (2) with a scalar potential $u^{i}=\nabla \phi^{i}(i=s, f)$, in the case of compression waves, and with a vector potential $u^{i}=\nabla \wedge \psi^{i}$ in the case of shear waves. The calculations are detailed in [1].

The results show that only two compression waves propagate simultaneously in the fluid phase and the solid phase of the porous material with elastic structure. The medium is then characterized by two wavenumbers $k_{1}$ and $k_{2}$ in (12) and four characteristic impedances $Z_{1}^{f}$ and $Z_{2}^{f}$ in (14) in 
the saturating fluid as well as $Z_{1}^{s}$ and $Z_{2}^{s}$ in (15) in the deformable matrix. Consider

$$
\begin{aligned}
& k_{1}^{2}=\frac{\omega^{2}}{2\left(P R-Q^{2}\right)}\left[P \widetilde{\rho}_{22}+R \widetilde{\rho}_{11}-2 Q \widetilde{\rho}_{12}-\sqrt{\nabla}\right], \\
& k_{2}^{2}=\frac{\omega^{2}}{2\left(P R-Q^{2}\right)}\left[P \widetilde{\rho}_{22}+R \widetilde{\rho}_{11}-2 Q \widetilde{\rho}_{12}+\sqrt{\nabla}\right],
\end{aligned}
$$

where

$$
\begin{aligned}
\nabla= & \left(P \widetilde{\rho}_{22}+R \widetilde{\rho}_{11}-2 Q \widetilde{\rho}_{12}\right)^{2} \\
& -4\left(P R-Q^{2}\left(\widetilde{\rho}_{11} \widetilde{\rho}_{22} \widetilde{\rho}_{12}\right)\right) .
\end{aligned}
$$

(i) In the fluid phase,

$$
\begin{aligned}
& Z_{1}^{f}=\left(R+\frac{Q}{\mu_{1}}\right) \frac{k_{1}}{\phi \omega}, \\
& Z_{2}^{f}=\left(R+\frac{Q}{\mu_{2}}\right) \frac{k_{2}}{\phi \omega} .
\end{aligned}
$$

(ii) In the solid phase,

$$
\begin{aligned}
& Z_{1}^{s}=\left(R+Q \mu_{1}\right) \frac{k_{1}}{\omega}, \\
& Z_{2}^{s}=\left(R+Q \mu_{2}\right) \frac{k_{2}}{\omega} .
\end{aligned}
$$

The reports $\mu_{1}$ and $\mu_{2}$ (16) between the speed in the solid frame and the speed in air, for the two compression waves, indicate in which medium, solid or fluid, the waves propagate preferentially:

$$
\mu_{i}=\frac{\phi_{i}^{f}}{\phi_{i}^{s}}=\frac{P k_{i}^{2}-\omega^{2} \widetilde{\rho}_{11}}{\omega^{2} \widetilde{\rho}_{11}-Q k_{i}^{2}} \quad i=1,2 .
$$

In contrast, only one shear wave propagates in the two mediums composing the porous material. These characteristics are given by

$$
\begin{aligned}
& k_{3}^{2}=\frac{\omega^{2}}{N}\left(\frac{\widetilde{\rho}_{11} \widetilde{\rho}_{22}-\widetilde{\rho}_{12}^{2}}{\widetilde{\rho}_{22}}\right), \\
& \mu_{3}=-\frac{\widetilde{\rho}_{12}}{\widetilde{\rho}_{22}} .
\end{aligned}
$$

In air at $18^{\circ} \mathrm{C}$, atmospheric pressure $P_{0}=1.0132 \times 10^{5} \mathrm{~Pa}$, with density of fluid $\rho_{0}=1.213 \mathrm{Kg} / \mathrm{m}^{3}$, sound wave speed $c_{0}=342.2 \mathrm{~m} / \mathrm{s}$, ration of specific heats $\gamma=1.4$, the Prandtl number $B^{2}=0.71$, and air viscosity $\eta=1.84 \times 10^{-5}[1]$.

\subsection{The $\left(u^{s}, p\right)$ Mixed Formulation for Poroelastic Material.} From Biot equations, Atalla et al. [16] have implanted an equivalent mixed formulation $\left(u^{s}, p\right)$. This formulation is valid only for harmonic oscillations. It is derived from the classic formulation which is equivalent mathematically.
The equilibrium modified equations (for small harmonic oscillations) are as follows:

$$
\begin{array}{r}
\nabla \widetilde{\sigma}^{s}\left(u^{s}\right)+\omega^{2} \widetilde{\rho} u^{s}+\widetilde{\gamma} \nabla p=0, \\
\nabla^{2} p+\frac{\omega^{2} \widetilde{\rho}_{22} p}{R}-\omega^{2}\left(\frac{\widetilde{\rho}_{22} \widetilde{\gamma}}{\phi^{2}}\right) \nabla u^{s}=0,
\end{array}
$$

where the tilde symbol $(\sim)$ indicates that the associated physical property is complex and frequency dependent. In (18), $\omega$ is the angular frequency; $u^{s}$ and $p$, respectively, denote the solid macroscopic displacement vector and the fluid sound pressure. $\widetilde{\sigma}^{s}$ denotes the modified partial stress tensor associated with the skeleton particle and only depends on the displacement of the solid phase. $\phi$ stands for the porosity defined as the ratio between the volume of the fluid phase and the total volume of the porous material, and $\widetilde{\rho}_{22}, \widetilde{\rho}_{12}$, and $\tilde{\rho}_{11}$ are given in the previous section. $\tilde{\rho}$ is the effective density given by $\tilde{\rho}=\widetilde{\rho}_{11}-\left(\tilde{\rho}_{12} / \widetilde{\rho}_{22}\right)$. The coefficient $\tilde{\gamma}$ is given by $\widetilde{\gamma}=\phi\left(\widetilde{\rho}_{12} / \widetilde{\rho}_{22}-Q / R\right)$

\section{Modeling Poroelastic Materials in COMSOL Multiphysics}

In this part, we will focus on the implementation of two equations from the Biot mixed formulation $\left(u^{s}, p\right)$ proposed by Atalla in COMSOL Multiphysics. We choose this formulation because it allows reducing the number of liberty degrees to four by a node instead of six liberty degrees when we use the classic Biot formulation. This gives a considerable reduction in calculation time. COMSOL is a tool for finite element analysis designed specifically to treat the multiphysics problems. The user combines a couple of predefined physics modules in COMSOL and introduces additional coupling terms to the constitutive equations. Of course, each physics mode can be used individually in the case of resolution of nonmultiphysics classic problems. Concerning porous materials, COMSOL does not provide a specific module. But, with the fact that these materials have a rigid structure and they can be modeled as equivalent fluid, the fluid dynamic module and acoustic module in COMSOL can be used. Moreover, porous materials have an elastic structure that can not be modeled multiphysically, that is, using solid mechanics and fluid mechanics modules to model solid and fluid phase, respectively. This is not only for not knowing the coupling terms but also for the high coupling in the equilibrium equations. As a result, the need of using EDP module is necessary to implement, for example, either the classic formulation or Biot mixed formulation, which are made in the form of differential equations. In COMSOL, the general form of PDE (for temporal analysis) must be expressed in the following matrix form:

$$
\Gamma \nabla=F,
$$

where $\Gamma$ is the flux vector matrix and $F$ is the right part of the vector (the two can be functions of spatial coordinates, the unknown variables $u$, and/or their derivatives in space), 
and $\nabla$ is the gradient/divergence operator. The dimensions of these quantities are as follows:

$$
\begin{aligned}
& \operatorname{dim} \Gamma=M \times N, \\
& \operatorname{dim} \nabla=N \times 1, \\
& \operatorname{dim} F=M \times 1 .
\end{aligned}
$$

Here $M$ is the number of equations equal to the unknowns number $(\operatorname{dim} u=M \times 1)$, whereas $N$ is the space dimension and then depends of the problem; it can be 1, 2, or 3 . In Cartesian coordinates, the gradient/divergence operator vector $\nabla$, for $N=3$, is defined as follows:

$$
\nabla=\left[\begin{array}{c}
\frac{\vartheta}{\vartheta x_{1}} \\
\frac{\vartheta}{\vartheta x_{2}} \\
\frac{\vartheta}{\vartheta x_{3}}
\end{array}\right] .
$$

The limit conditions in the case of PDE in the general form are as follows:

$$
\begin{aligned}
0 & =R, \\
-\Gamma n & =G+\left[\frac{\vartheta R}{\vartheta u}\right]^{T} \mu,
\end{aligned}
$$

where the vector $R$ and $\Gamma$ can be functions of space coordinates, the unknown variable $u$, and/or their spatial derivatives, whereas $n$ is the normal unit vector outgoing from the limit surface. These are, respectively, the limit conditions of Dirichlet and Neumann. The term $\mu$ in the Neumann limit condition is a synonym of Lagrange multiplier. To analyze the harmonic behavior of a porous medium, we use the mixed formulation of Biot (18). The latter depends on 4 variables $(M=4)$ : the displacements of the solid phase, $u_{i}$, and the fluid pressure, $p$. From these two equations, the matrices $\Gamma$ and $F$, constituting the form (19), are identified as $[14,15,19]$

$$
\begin{aligned}
& \Gamma=\left[\begin{array}{c}
\Gamma_{i j} \\
\Gamma_{4 j}
\end{array}\right]=\left[\begin{array}{c}
\widehat{\sigma}^{s}\left(u^{s}\right) \\
\nabla p
\end{array}\right], \\
& F=\left[\begin{array}{c}
F_{i} \\
F_{4}
\end{array}\right]=\left[\begin{array}{c}
-\omega^{2} \widetilde{\rho} u^{s}-\widetilde{\gamma} \nabla p \\
-\frac{\omega^{2} \widetilde{\rho}_{22} p}{R}+\omega^{2}\left(\frac{\widetilde{\rho}_{22} \widetilde{\Gamma}}{\phi^{2}}\right) \nabla u^{s}
\end{array}\right] .
\end{aligned}
$$

According to the definition in [16], the expression of $\widetilde{\sigma}^{s}\left(u^{s}\right)$ can be written as

$$
\widetilde{\sigma}\left(u^{s}\right)=\left(A-\frac{Q^{2}}{R}\right) \nabla u^{s}+2 N \epsilon^{s},
$$

where $A$ is the Lamé coefficient for the elastic solid.
Define

$$
u_{k, k}^{s}=\nabla u^{s}=\frac{\partial u}{\partial x}+\frac{\partial v}{\partial y}+\frac{\partial w}{\partial x}
$$

$\Gamma$ and $F$ can be rewritten in detail as

$\Gamma$

$$
\begin{aligned}
= & {\left[\begin{array}{ccc}
2 N u_{x}+\widetilde{A} u_{k, k}^{s} & N\left(u_{y}+v_{x}\right) & N\left(u_{z}+w_{x}\right) \\
N\left(u_{y}+v_{x}\right) & 2 N v_{y}+\widetilde{A} u_{k, k}^{s} & N\left(w_{y}+v_{z}\right) \\
N\left(u_{z}+w_{x}\right) & N\left(v_{z}+w_{y}\right) & 2 N w_{z}+\widetilde{A} u_{k, k}^{s} \\
p_{x} & p_{y} & p_{z}
\end{array}\right], } \\
F & =\left[\begin{array}{c}
-\omega^{2} \tilde{\rho} u-\tilde{\gamma} p_{x} \\
-\omega^{2} \tilde{\rho} v-\tilde{\gamma} p_{y} \\
-\omega^{2} \tilde{\rho} w-\tilde{\gamma} p_{z} \\
-\frac{\omega^{2} \tilde{\rho}_{22} p}{R}+\frac{\omega^{2} \tilde{\gamma} \tilde{\rho}_{22} u_{k, k}^{s}}{\phi^{2}}
\end{array}\right] .
\end{aligned}
$$

3.1. Poroelastic/Air Coupling. In the case of poroelastic medium bound to an acoustic medium, (27) describes the continuity conditions of the total normal stress, acoustic pressure, and fluid flow. Consider

$$
\begin{aligned}
\sigma^{t} n & =-p^{a} n, \\
P & =p^{a}, \\
(1-\phi) u^{s} n+\phi u^{f} n & =\left(\rho_{0} \omega^{2}\right)^{-1} \nabla p^{a} n .
\end{aligned}
$$

Here, $p^{a}$ is the pressure in the acoustic medium, $\sigma^{t}$ is the total stress tensor in the poroelastic material, $u^{f}$ is the displacement of the fluid phase in the $\left(u^{s}, u^{f}\right)$ formulation, and $n$ is the outward normal unit vector. The detailed expressions for $u^{f}$ and $\sigma^{t}$ were given by Atalla et al. [16]. After some substitution, the vectors $G$ and $R$ can be expressed as

$$
\begin{aligned}
G & =\left[\begin{array}{c}
{\left[1-\phi\left(1+\frac{Q}{R}\right)\right] p^{a} n_{x}} \\
{\left[1-\phi\left(1+\frac{Q}{R}\right)\right] p^{a} n_{y}} \\
{\left[1-\phi\left(1+\frac{Q}{R}\right)\right] p^{a} n_{z}} \\
0
\end{array}\right], \\
R & =\left[\begin{array}{c}
0 \\
0 \\
0 \\
p-p^{a}
\end{array}\right] .
\end{aligned}
$$

\section{Acoustic Absorption Coefficient}

In this section, we consider a structure composed of a poroelastic medium glued on a rigid wall (the rigid wall is 
TABLE 1: Poroelastic properties of polyurethane foam.

(a)

\begin{tabular}{lccccc}
\hline Porosity & Resistivity & Tortuosity & VCL & TCL & \multicolumn{2}{c}{ Density skeleton } \\
$\phi$ & $\sigma$ & $\alpha_{\infty}$ & $\wedge$ & $\wedge^{\prime}$ & $\rho_{0}$ \\
\hline 0.97 & $87 \mathrm{KN} \mathrm{s} / \mathrm{m}^{4}$ & 2.52 & $37 * 10^{-6} \mathrm{~m}$ & $119 * 10^{-6} \mathrm{~m}$ & $31 \mathrm{Kg} / \mathrm{m}^{3}$ \\
\hline
\end{tabular}

(b)

\begin{tabular}{lcr}
\hline Loss factor & Poisson's coefficient & Shear modulus \\
$\eta$ & $v$ & $N$ \\
\hline 0.055 & 0.3 & $55(1+v j) \mathrm{KPa}$ \\
\hline
\end{tabular}

VCL: viscous characteristic length.

TCL: thermal characteristic length.

a condition to the rear boundary); the surface impedance of this structure was introduced by Allard [1] and given by

$$
\begin{aligned}
Z_{s}= & -j \frac{Z_{1}^{s} Z_{2}^{f} \mu_{2}-Z_{2}^{s} Z_{1}^{f} \mu_{1}}{D_{m r}}, \\
D_{m r} & \\
= & \left(1-\phi+\phi \mu_{2}\right)\left[Z_{1}^{s}-(1-\phi) Z_{1}^{f} \mu_{1}\right] \tan \left(k_{2} e\right) \\
& +\left(1-\phi+\phi \mu_{1}\right)\left[Z_{2}^{s} \mu_{2}-(1-\phi) Z_{1}^{f}\right] \tan \left(k_{1} e\right) .
\end{aligned}
$$

$Z_{1}^{s}, Z_{2}^{s}$ and $Z_{1}^{f}, Z_{2}^{f}$ are the characteristic impedances of the poroelastic medium and the fluid, respectively [1]. $\mu_{1}, \mu_{2}$ are the ratios between the speeds in poroelastic material and fluid, respectively [1]. However, in COMSOL environment, $Z^{s}$ is defined as the ratio of the acoustic pressure and the total velocity at the impinged face [20], and it can be written as

$$
\begin{aligned}
& Z_{s}(\omega)=\frac{p}{j \omega\left(\phi u_{3}^{f}+(1-\phi) u_{3}^{s}\right)} \\
& \quad=p\left(j \omega\left[\frac{\phi}{\omega^{2} \widetilde{\rho}_{22}} p_{z}+\left(1-\phi\left(1+\frac{\tilde{\rho}_{12}}{\widetilde{\rho}_{22}}\right)\right) \omega\right]\right)^{-1} .
\end{aligned}
$$

The sound absorption of a poroelastic layer glued to a rigid wall and submitted to plane acoustic wave propagating in the air at the surface of the layer at normal incidence is calculated from the surface impedance $Z_{s}$ and the impedance of air as follows $[1,7,21]$ :

$$
\alpha_{\infty}=1-\left|\frac{Z_{s}-Z_{0}}{Z_{s}+Z_{0}}\right|^{2} \quad \text { with } Z_{0}=\rho_{0} c_{0}
$$

\section{Numerical Results and Discussion}

The acoustic properties of polyurethane foams are given in Table 1 [22], the thickness of this foam is $16 \mathrm{~mm}$, and one of its extremities is glued to a rigid wall, while the other is excited with a normal incidence by a monochromatic pressure wave of pulsation $\omega$. We have calculated analytically the real and imaginary parts of surface impedance, shown in Figures 1 and 2, and the absorption coefficient versus frequency

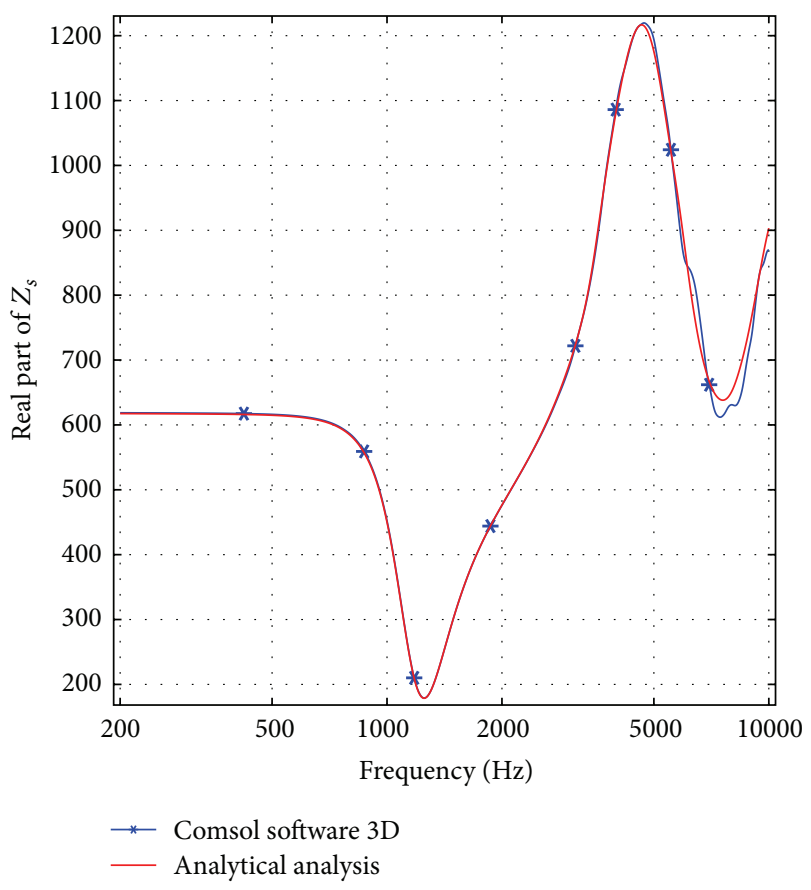

FIGURE 1: Real part of surface impedance.

(Figure 3). In order to validate the obtained results we made a comparison with results calculated with COMSOL in $3 \mathrm{D}$. Indeed, we found an excellent agreement between the results calculated by the two methods. Since the foam skeleton is set to motion, which depends on the frequency, the study of the absorption coefficient is made in three characteristic frequency bands centered around the resonance frequency (32) of the skeleton, which is the quart-wave frequency for the materials glued to a rigid wall. In the vicinity of this frequency, the rigidity of the frame can have a huge influence on the absorption coefficient. The foam fluid phase (air) is very light and has compressibility modulus much lesser than the skeleton's, which allows the estimation of this frequency by simply considering the properties of the frame under vacuum as follows [23]:

$$
f_{r} \approx \frac{1}{4 e} \sqrt{\frac{E((1-v) /(1+v)(1-2 v))}{\rho_{s}}},
$$




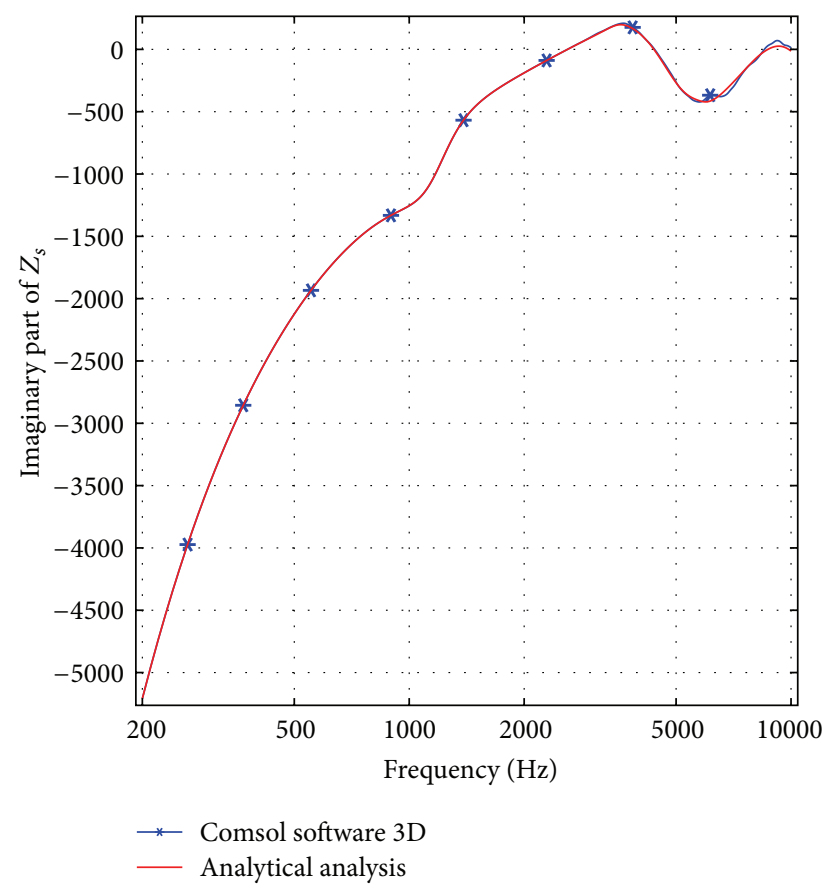

FIGURE 2: Imaginary part of surface impedance.

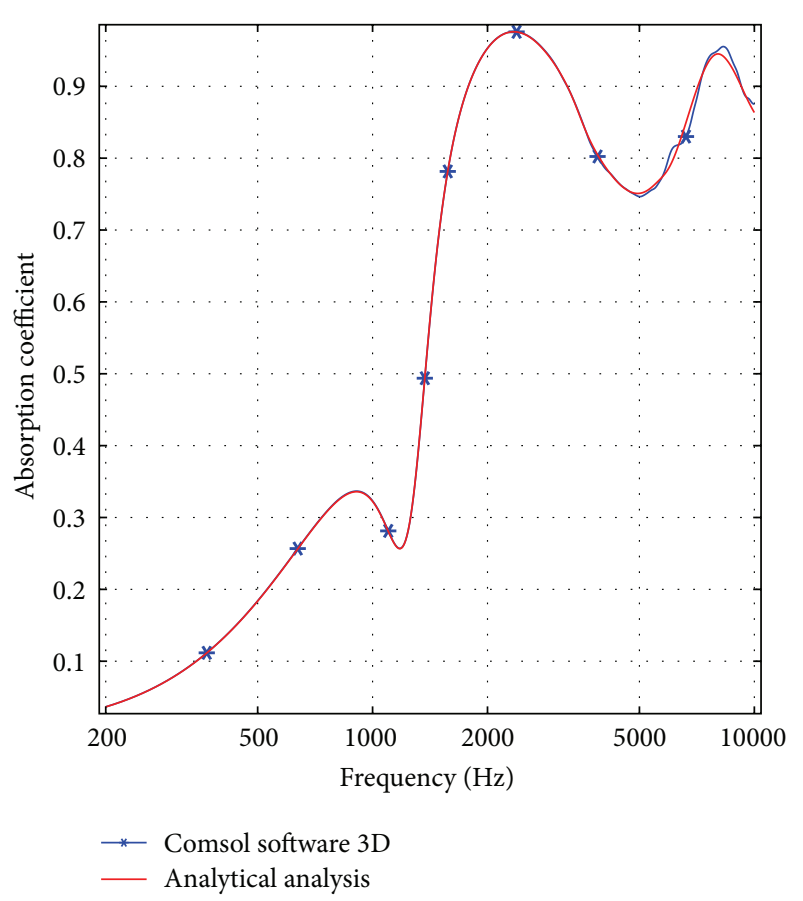

FIgURE 3: Absorption coefficient of polyurethane foam.

where $e$ is the thickness of the porous layer (here $e=$ $16 \mathrm{~mm}) . \rho_{s}$ is the skeleton density (i.e., the density of the porous material in vacuum). The estimation of quart wave resonance frequency for the layer made of this foam is $f_{r}=1.23 \mathrm{kHz}$. From this frequency, we can find three characteristic zones, the low frequencies range $[\mathrm{LF}]\left[1, f_{r} / 2\right]$, the medium frequencies range $[\mathrm{MF}]\left[f_{r} / 2,2 f_{r}\right]$, and the high frequencies range $[\mathrm{HF}]\left[2 f_{r}, 10^{4}\right]$. In Figure 3 , the low frequencies zone (between 1 and $615 \mathrm{~Hz}$ ) and medium frequencies (between 615 and $2462 \mathrm{~Hz}$ ), small absorption has been observed, while, in the high frequencies zone (between 2462 and $10^{4} \mathrm{~Hz}$ ), the absorption coefficient gets a maximal value for certain frequencies and drops slightly each time reaching the maximum.

5.1. Mechanical Behavior of Porous Materials. The skeleton of porous materials generally has a mechanical behavior of a viscoelastic type at room temperature and in the audible frequency range $20 \mathrm{~Hz}$ to $20 \mathrm{kHz}$. Therefore, its response to mechanical stress depends on the time or the stress frequency (or pulse $\omega$ ) and also the temperature; the two variables are closely linked: the apparent stiffness of the polymer decreases with increasing temperature or as the frequency decreases. As part of this paper, the polymeric foam (polyurethane) will be subject to normal conditions of temperature and pressure $\left(T_{0}\right.$ $=18^{\circ} \mathrm{C}$ and $P_{0}=1.0132105 \mathrm{~Pa}$ ).

In the case of small deformations, the behavior can be considered linear and described by Hooke's law using complex variables [24]. Using a vector representation of strain and stress field, we have

$$
\left\{\widetilde{\sigma}_{s}\right\}=\left[\widetilde{H}_{E}\right]\left\{\widetilde{\epsilon}^{s}\right\},
$$

where $\widetilde{\sigma}_{s}$, $\widetilde{\epsilon}^{s}$, and $\widetilde{H}_{E}$, respectively, are the stress, strain, and the complex matrix of elasticity $E$ of the solid phase (the tilde symbol for a complex and frequency-dependent quantity). In the case of an isotropic model, the elastic matrix $\widetilde{H}_{E}$ can be characterized from the Young modulus and the complex Poisson coefficient. Consider

$$
\begin{aligned}
& \widetilde{E}=E(\omega)+j E^{\prime}(\omega)=E(\omega)(1+j \eta(\omega)), \\
& \widetilde{\nu}=\nu(\omega)+j v^{\prime}(\omega)
\end{aligned}
$$

where $\eta$ is the loss factor defined as the ratio of the instantaneous response (real part of the matrix) on the quadrature phase response (imaginary part). If we consider that the skeleton is isotropic-transverse, in this case, five elastic coefficient are necessary $[25,26] . \widetilde{E}_{L}$ and $\widetilde{E}_{T}$ are, respectively, the moduli of elasticity in the longitudinal and transverse directions. $\widetilde{G}_{L T}$ is shear modulus in the plane $(z, y)$. $\widetilde{v}_{L T}$ is the Poisson coefficient proportional to the deformation in the transverse plane generated by a deformation in the longitudinal plane, and $\widetilde{\nu}_{T T}$ is the Poisson coefficient in the transverse plane.

Because of the difficulty of determining the above five parameters and the real directions of symmetry, we will focus in this paper on an isotropic model. This assumption allows us the use of an analytical model to describe the vibroacoustic behavior of the porous material. In addition, Poisson's coefficient is considered real and independent of frequency $\widetilde{v}=v$ [27]: this is fixed to 0.3 for polymers foams 


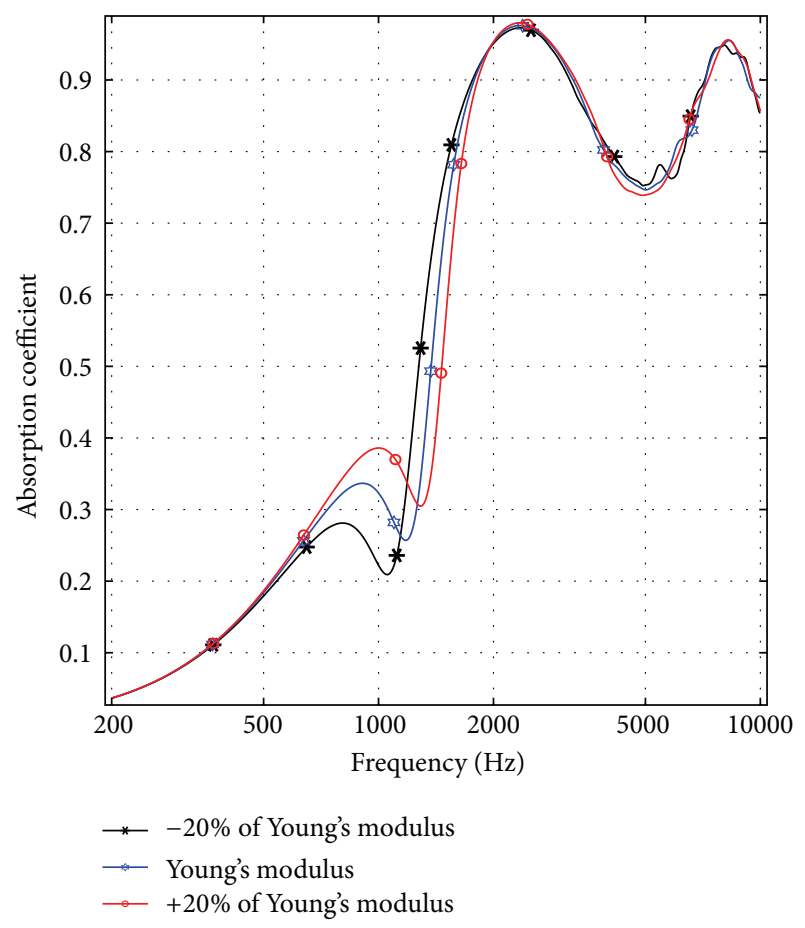

FIgURE 4: $E$ effects on absorption coefficient.

such as polyurethane. So, from this assumption, (34) are written as follows:

$$
\begin{aligned}
\widetilde{E} & =E(\omega)(1+j \eta(\omega)), \\
\widetilde{\nu} & =\nu(\omega) .
\end{aligned}
$$

5.2. Influence of the Mechanical Parameters on the Acoustic Absorption. In this part, we discuss the importance of the structure vibration of the polyurethane foam on its absorption properties and study the mechanical parameters effects on these properties (i.e., the Young modulus $E$, structural loss coefficient $\eta$, the Poisson coefficient $\nu$, and the density $\rho_{1}$ ). Figure 4 shows the variations of the absorption coefficient by varying the Young modulus by $\pm 20 \%$; we notice that this variation is found around the frequency of resonance given by (32); when the value of the Young modulus increases or decreases by $\pm 20 \%$, the maximum amplitude of resonance increases and decreases with a value of 0.05 , we also notice that there is a shift in frequency of this maximum of a value of $100 \mathrm{~Hz}$ towards the higher frequencies in the case where the Young modulus increases and towards the lower frequencies when it decreases, without any modification in the quality factor (Figure 5).

In the same way concerning the influence of the Poisson coefficient on the absorption, the same variation has been observed (Figure 6) except there is a small increase in the resonance amplitude as the value of the Poisson coefficient increases by $20 \%$ (Figure 7 ).

In Figure 8, a variation of 0.02 of the resonance amplitude has been observed; a decrease or an increase occurs depending on the variation in the value of the material density, with

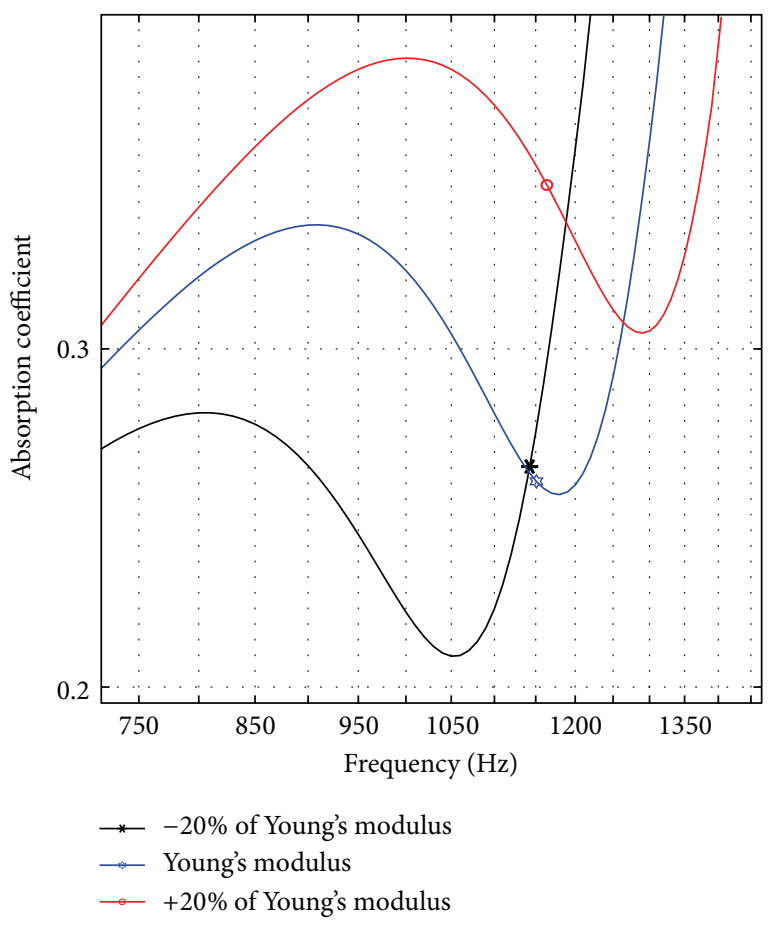

FIGURE 5: The zoom around of $f_{r}$.

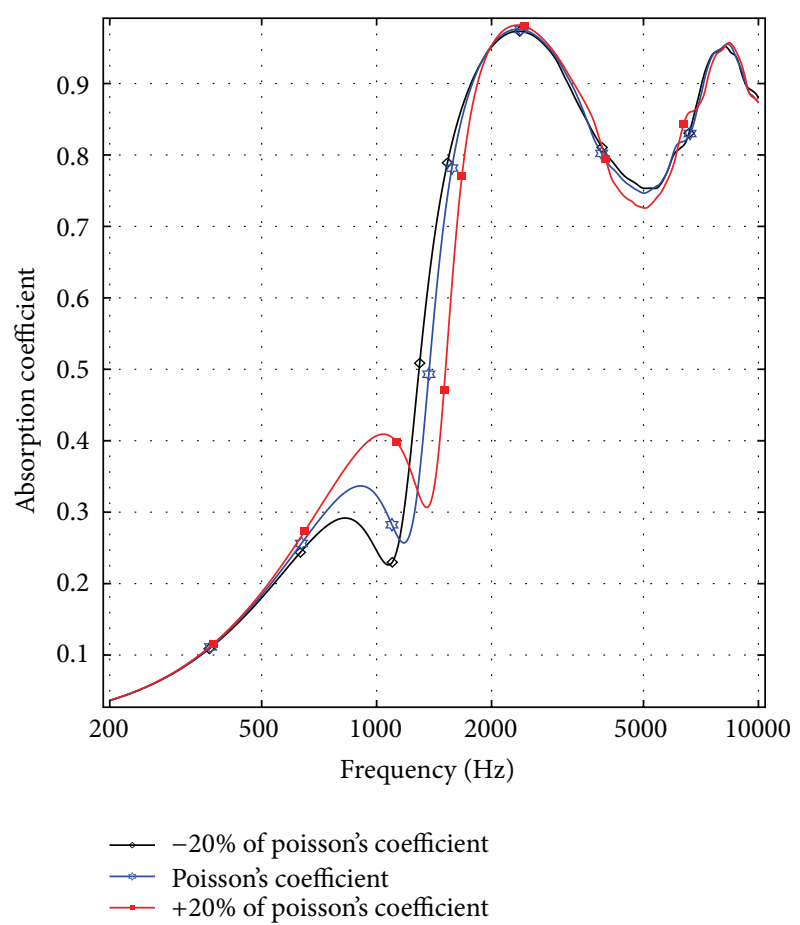

FIGURE 6: $v$ effects on absorption coefficient.

a resonance frequency shift of $50 \mathrm{~Hz}$ (Figure 9), while, in Figure 10, we distinguish a small variation observed at level of the resonance amplitude without any shift in the resonance frequency (Figure 11). 


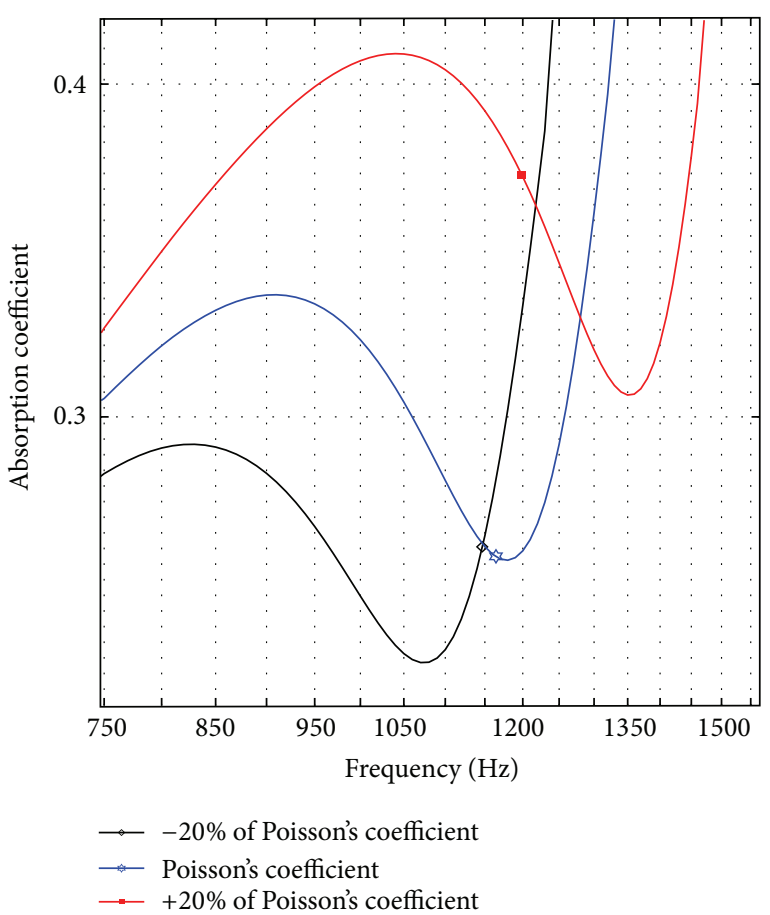

FIGURE 7: The zoom around of $f_{r}$.

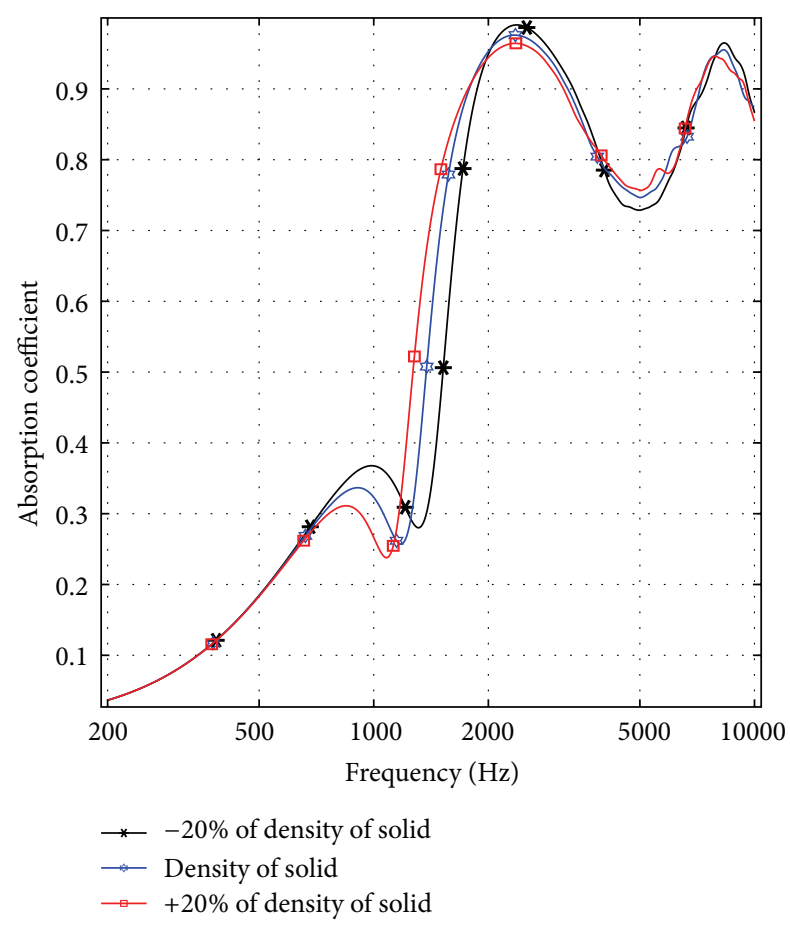

FIGURE 8: $\rho_{1}$ effects on absorption coefficient.

\section{Conclusion}

In this paper, we presented a study of the efficiency of acoustic absorption in poroelastic medium of type polymer foam (a case of polyurethane foam). Using the generalized BiotAllard model and combined formulation of Atalla et al.,

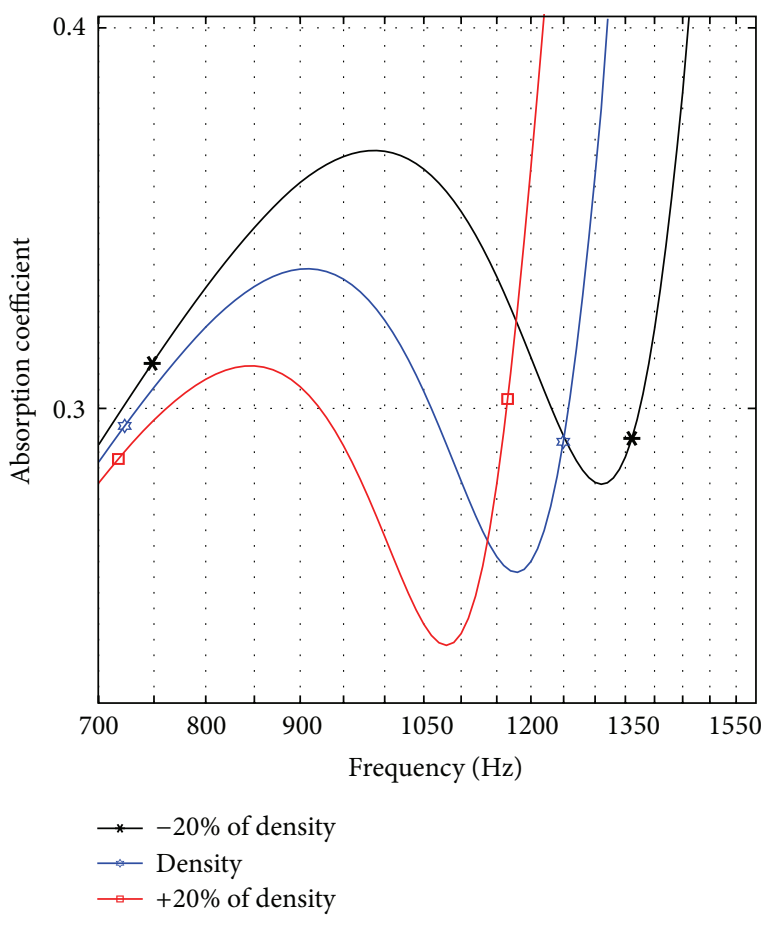

FIGURE 9: The zoom around of $f_{r}$.

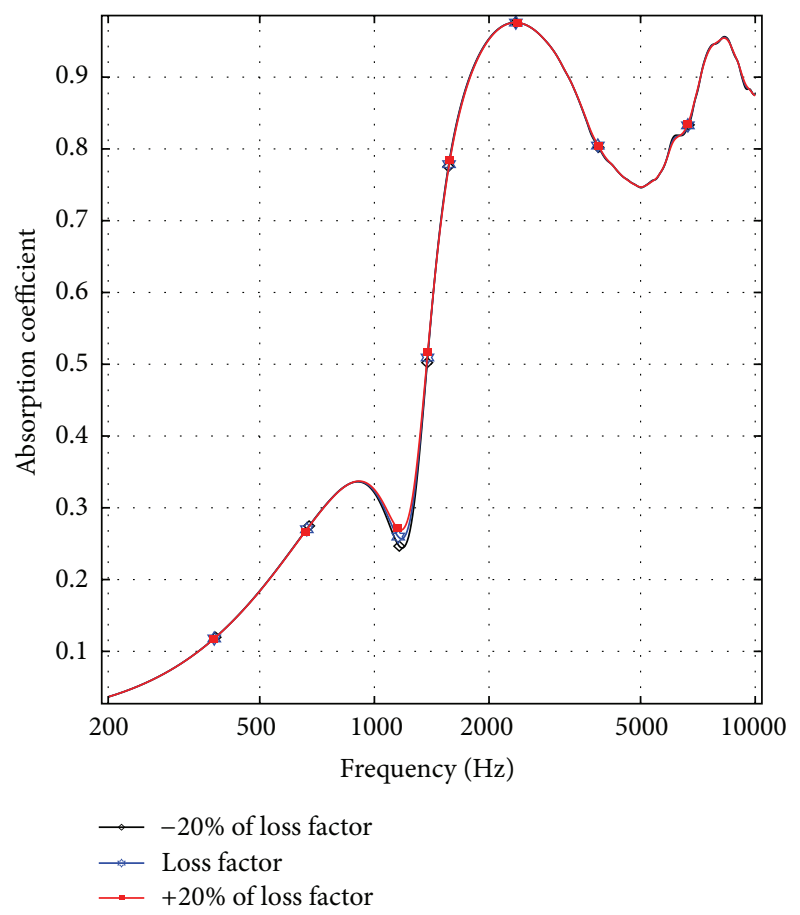

FIgURE 10: $\eta$ effects on absorption coefficient.

the real and imaginary parts were calculated. The obtained results show an excellent agreement between analytical calculations and those numericals performed using COMSOL environment. These results confirm that the foam has a very good performance in absorbing noise at medium and 


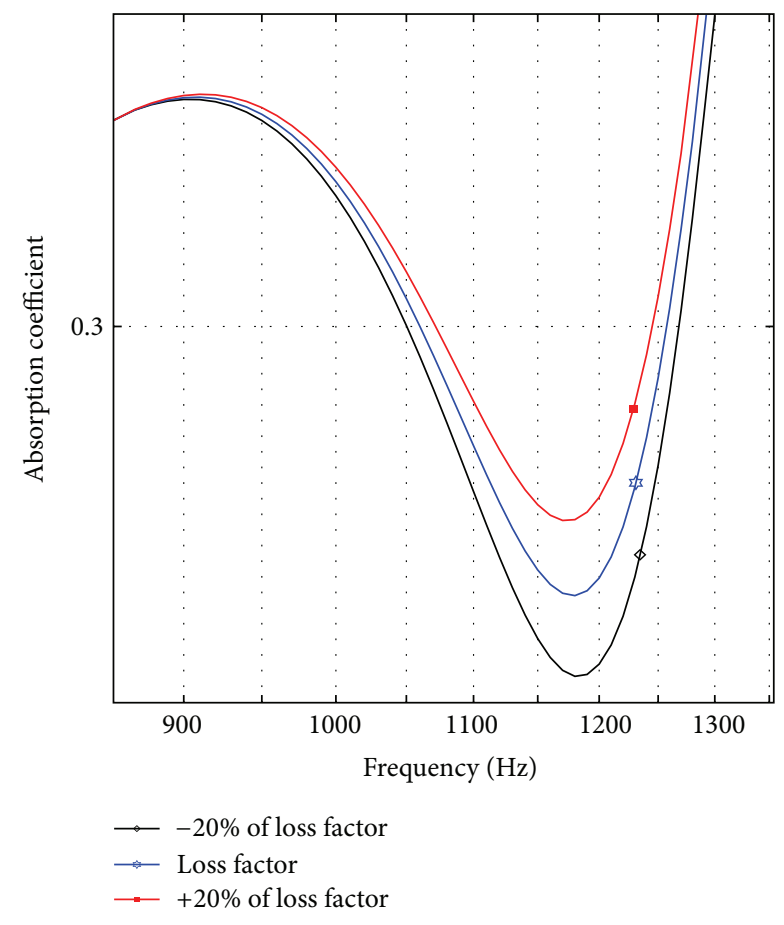

FIGURE 11: The zoom around of $f_{r}$.

high frequencies and less performance in low frequencies. Afterwards, simulations have been done in COMSOL Multiphysics to study the effect of mechanical parameters such as the Young modulus E, the Poisson coefficient $v$, structural damping factor $\eta$, and density of material $\rho_{1}$ on the absorption coefficient. The simulations showed that the Young modulus, the Poisson coefficient, and the density of material play an important role in the absorption quality of the polyurethane foam particularly around the resonant frequency, while the structural damping factor plays a minor role in the acoustic absorption. Determining experimentally the mechanical parameters is needed to better understand and predict the acoustic behavior of this foam.

\section{Conflict of Interests}

The authors declare that there is no conflict of interests regarding the publication of this paper.

\section{References}

[1] J.-F. Allard, Propagation of Sound in Porous Media: Modeling Sound Absorbing Materials, Applied Science, Elsevier, 1993.

[2] K. Attenborough, "Models for the acoustical characteristics of air filled granular materials," Acta Acustica, vol. 1, pp. 213-226, 1993.

[3] D. L. Johnson, J. Koplik, and R. Dashen, "Theory of dynamic permeability and tortuosity in fluid-saturated porous media," Journal of Applied Physics, vol. 176, pp. 379-402, 1991.

[4] Y. Champoux and J.-F. Allard, "Dynamic tortuosity and bulk modulus in air-saturated porous media," Journal of Applied Physics, vol. 70, no. 4, pp. 1975-1979, 1991.
[5] D. K. Wilson, "Simple, relaxational models for the acoustical properties of porous media," Applied Acoustics, vol. 50, no. 3, pp. 171-188, 1997.

[6] S. R. Pride, F. D. Morgan, and A. F. Gangi, "Drag forces of porous-medium acoustics," Physical Review B, vol. 47, no. 9, pp. 4964-4978, 1993.

[7] B. Brouard, D. Lafarge, and J.-F. Allard, "A general method of modelling sound propagation in layered media," Journal of Sound and Vibration, vol. 183, no. 1, pp. 129-142, 1995.

[8] M. A. Biot, "Theory of elasticity and consolidation for a porous anisotropic solid," Journal of Applied Physics, vol. 26, no. 2, pp. 182-185, 1955.

[9] M. A. Biot, "Theory of propagation of elastic waves in a fluidsaturated porous solid. I. Low-frequency range," The Journal of the Acoustical Society of America, vol. 28, no. 2, pp. 168-178, 1956.

[10] M. A. Biot, "The theory of propagation of elastic waves in a fluidsaturated porous solid. II. High frequency range," The Journal of the Acoustical Society of America, vol. 28, pp. 179-191, 1956.

[11] M. Ouisse, M. Ichchou, S. Chedly, and M. Collet, "On the sensitivity analysis of porous material models," Journal of Sound and Vibration, vol. 331, no. 24, pp. 5292-5308, 2012.

[12] F. Scarpa and F. C. Smith, "Passive and MR fluid-coated auxetic PU foam-mechanical, acoustic, and electromagnetic properties," Journal of Intelligent Material Systems and Structures, vol. 15, no. 12, pp. 973-979, 2004.

[13] O. Doutres, N. Dauchez, J.-M. Génevaux, and O. Dazel, "A frequency independent criterion for describing sound absorbing materials by a limp frame model," Acta Acustica united with Acustica, vol. 95, no. 1, pp. 178-181, 2009.

[14] C. Batifol, T. G. Zielinski, M. N. Ichchou, and M.-A. Galland, "A finite element study of a piezoelectric/poroelastic sound package concept," Smart Materials and Structures, vol. 16, pp. 168-177, 2007.

[15] T. G. Zielinski, "Modeling and analysis of smart technologies in vibroacoustics," in Smart Technologies for Safety Engineering, chapter 8, John Wiley \& Sons, Chichester, UK, 2008.

[16] N. Atalla, R. Panneton, and P. Debergue, "A mixed displacement-pressure formulation for poroelastic materials," Journal of the Acoustical Society of America, vol. 104, no. 3, pp. 1444-1452, 1998.

[17] N. Atalla and J. F. Allard, Propagation of Sound in Porous Media: Modelling Sound Absorbing Materials, Wiley, 2nd edition, 2009.

[18] O. Hilbrunner, Caractérisation des matériaux visco-poroélastiques à usage vibro-acoustique [DEA thesis], Central School of Lyon, 2000.

[19] C. Batifol, T. G. Zielinski, M.-A. Galland, and M. N. Ichchou, "Hybrid piezo-poroelastic sound package concept: numeri$\mathrm{cal} /$ experimental validations," in Proceedings of the International Symposium on Active Control and Vibration (Active '06), Adelaide, Australia, 2006.

[20] Y. Hu, M. A. Galland, and K. Chen, "Acoustic transmission performance of double-wall active sound packages in a tube: numerical/experimental validations," Applied Acoustics, vol. 73, no. 4, pp. 323-337, 2012.

[21] C. Zhao, P. Wang, L. Wang, and D. Liu, "Reducing railway noise with porous sound-absorbing concrete slabs," Advances in Materials Science and Engineering, vol. 2014, Article ID 206549, 11 pages, 2014.

[22] T. G. Zieliński, "Numerical investigation of active porous composites with enhanced acoustic absorption," Journal of Sound and Vibration, vol. 330, no. 22, pp. 5292-5308, 2011. 
[23] B. Castagnéde, A. Aknine, M. Melon, and C. Depollier, "Ultrasonic characterization of the anisotropic behavior of airsaturated porous materials," Ultrasonics, vol. 36, no. 1-5, pp. 323-341, 1998.

[24] M. Etchessahar, "Caractérisation mécanique en basses fréquences des matériaux acoustiques," 2002.

[25] S. Sahraoui and E. Mariez, "Elastic constants of polyurethane foam's skeleton for biot model," in Proceedings of the International Congress on Noise Control Engineering (Internoise'96), pp. 951-954, 1996.

[26] S. Sahraoui, E. Mariez, and M. Etchessahar, "Linear elastic properties of anisotropic open-cell foams," Journal of the Acoustical Society of America, vol. 110, no. 1, pp. 635-637, 2001.

[27] T. Pritz, "Measurement methods of complex poisson's ratio of viscoelastic materials," Applied Acoustics, vol. 60, no. 3, pp. 279292, 2000. 

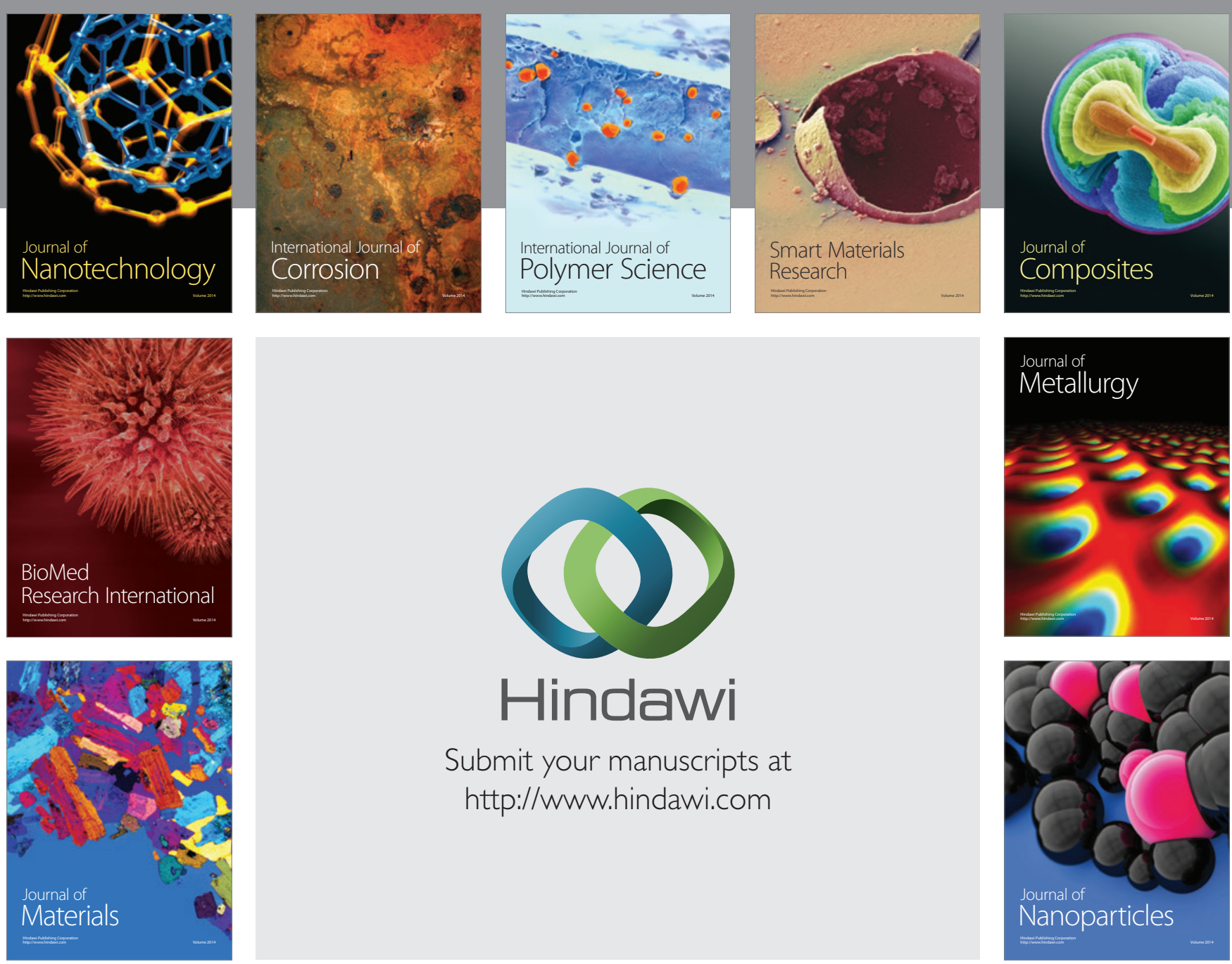

Submit your manuscripts at http://www.hindawi.com
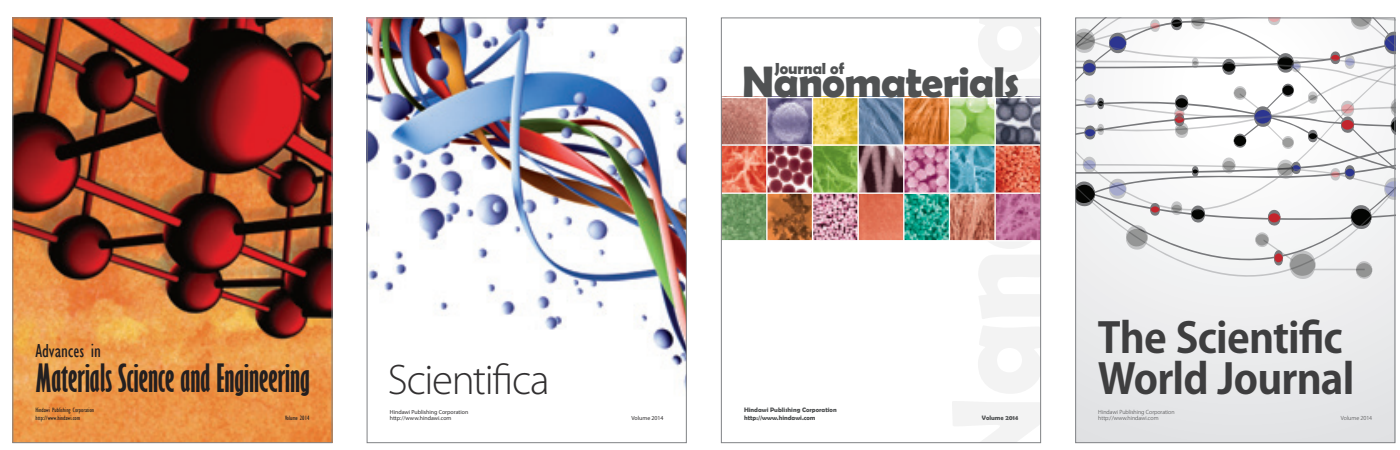

\section{The Scientific World Journal}
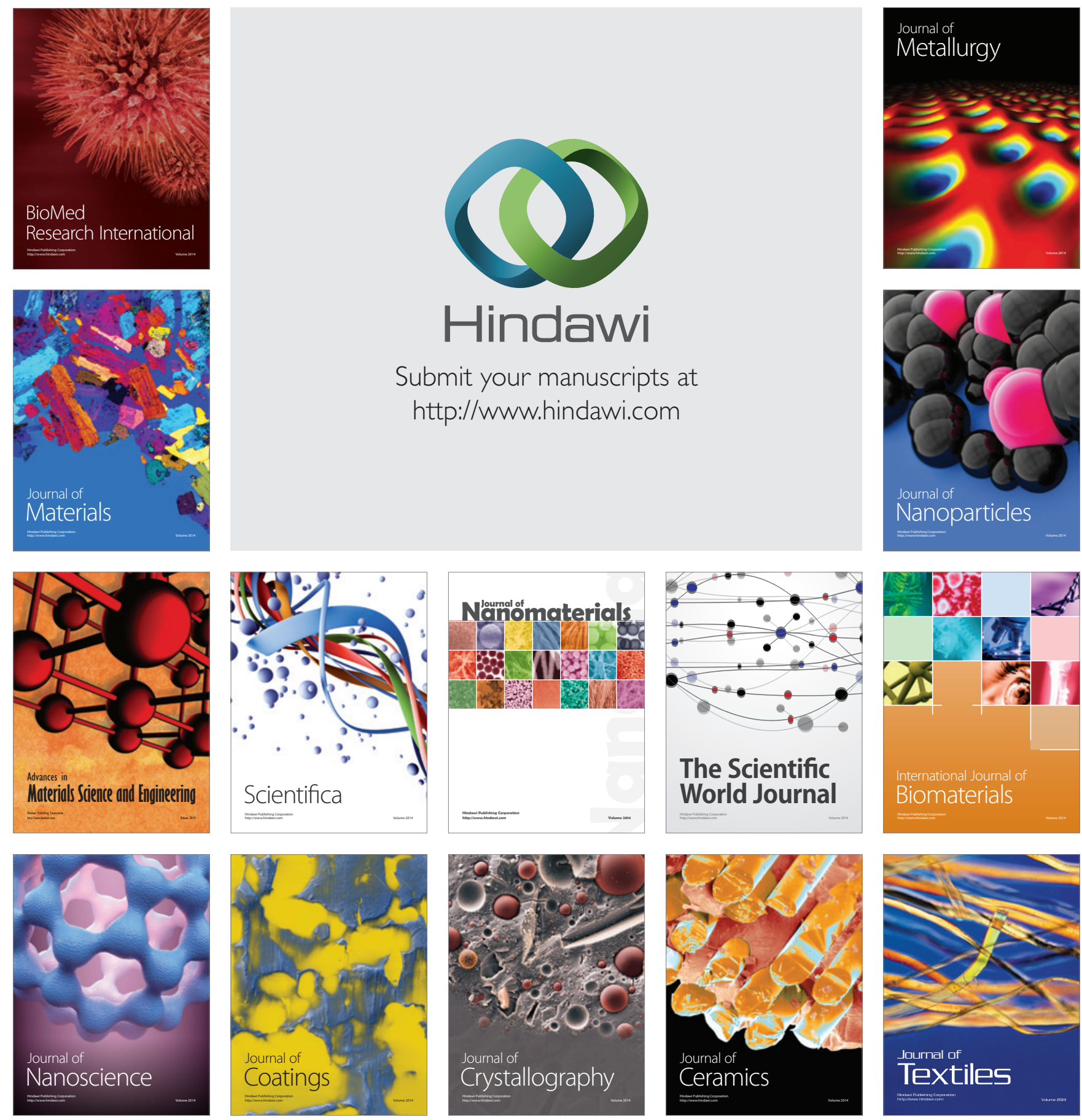